\title{
Para-Infectious Acute Transverse Myelitis Following Sars-Cov2 Infection: A Case Report
}

\author{
Oana Mosora1, Valentin Moroșanu¹, Adina Stoian ${ }^{1,2^{*}}$, Rodica Bălașa1,2 \\ 1. Neurology 1 Clinic, Emergency Clinical County Hospital Mures, Targu Mures, Romania \\ 2. George Emil Palade University of Medicine, Pharmacy, Science, and Technology of Targu Mures, Romania
}

Introduction: The new pandemic has highlighted new ways of clinical presentation of severe acute respiratory syndrome coronavirus 2 (SARS-COV-2) including a possible link to central nervous system (CNS) manifestation. Case report: We present a case of acute transverse myelitis following a SARS-CoV 2 infection in a 34-year-old man who presented with bilateral lower-extremity weakness and acute urinary retention. Cervical magnetic resonance imaging showed a T2 hyperintense signal abnormality at C3-C6 and D3-D7 levels consistent with acute myelitis. Conclusion: SARS-CoV 2 can cause myelitis by immune-mediated mechanisms, therefore it is extremely important for the clinicians to recognise the signs and promptly treat this neurological complication.

Keywords: SARS-CoV2, para-infectious acute transverse myelitis, central nervous system

Received 3 September 2021 / Accepted 10 September 2021

\section{Introduction}

At the end of December 2019, an outbreak of atypical pneumonia caused by a novel severe acute respiratory syndrome coronavirus-2 (SARS-CoV-2), was reported from Wuhan, China [1]. Although, the most common presentation is with respiratory symptoms, there is increasing evidence that the nervous system is frequently affected by the virus [2]. The mechanism for viral entry into the central nervous system (CNS) is presumed to be via the olfactory nerve or other cranial or peripheral nerves (trigeminal nerve, vagus nerve), lymphatic spread or entry through the choroid plexus [3]. The COVID-19 virus could also enter the cells by angiotensin-converting enzyme 2 - receptor (ACE2-R) pathways (ACE). In the CNS, ACE2-R is expressed in neurons, astrocytes, oligodendrocytes, and is concentrated in posterior cingulate cortex, substantia nigra, middle temporal gyrus and the olfactory bulb [4]. ACE2- $\mathrm{R}$ is a far-reaching target for influenza viruses, as well as CoV. Furthermore, the interaction between SARS$\mathrm{CoV}-2$ spike protein and ACE2-R could result in bloodbrain barrier disruption and dissemination into the CNS [5].

SARS-CoV-2 can induce neuronal injury by multiple mechanisms such as cytokine storm associated with hyperinflammatory syndrome a para- or post-infectious inflammatory disease, an immune-mediated disease or by augmenting a systemic disorder (sepsis, hypoxia, hypercoagulability) [6].

A broad spectrum of neurological complications such as ischemic stroke, acute demyelinating polyradiculoneuritis, acute encephalitis and meningoencephalitis, acute necrotizing encephalopathy, acute transverse myelitis have been reported [7].

* Correspondence to: Adina Stoian Email: cretadina@yahoo.com
Transverse myelitis is an inflammatory myelopathy that can lead to disabling neurological deficits such as motor weakness and sensory deficits, as well as autonomic dysfunction (bladder, bowel and sexual) [8]. Several aetiologies have been incriminated for acute transverse myelitis: paraneoplastic myelitis, drug-induced myelitis, myelitis appearing in the context of various systemic autoimmune disorders (lupus erythematosus, Sjogren syndrome) and demyelinating diseases such as multiple sclerosis (MS) and neuromyelitis optica (NMO), post-radiation myelitis , post-vaccination and post-infectious myelitis. It is extremely important to recognize the diagnosis of transverse myelitis as it is associated with significant morbidity and mortality [9].

\section{Case report}

A 34 year-old male with no significant prior medical history presented to the local emergency department with paresthesias (numbness and tingling) in the lower extremities and lower limbs weakness. Two weeks before the admission he tested positive for SARS-CoV-2. The patient did not present with respiratory distress, had no fever or altered general state, apart from hyposmia and dysgeusia. He did not receive any specific treatment for COVID-19 and hospitalization was not required.

Neurological examination revealed preserved cranial nerve functions, flaccid paraparesis grade 3/5 Medical Research Council (MRC), decreased sensation at D6 level, decreased vibration sensation in the lower limbs, positive bilateral Babinski sign and urinary retention that required urinary catheterisation

Initial laboratory results showed elevated inflammatory markers: Erythrocyte Sedimentation Rate (ESR), fibrinogen, a slightly elevated white blood cell count $\left(11.7^{\wedge} 3 /\right.$ $\mathrm{UL}$ ), high value for D-Dimer (403ng/dl). Autoimmune immunological screening was negative for antinuclear an- 
tibodies (ANA), anti-double stranded DNA antibodies, anticardiolipin antibodies. Serological investigations for infectious diseases (Venereal Disease Research Laboratory test, HIV I and II, hepatitis B and C, Epstein Barr, Borrelia burgdorferi) were negative. Testing for aquaporin-4 and oligoclonal bands was negative, however myelin oligodendrocyte glycoprotein (MOG) antibodies were positive. Cerebrospinal fluid analysis (CSF) showed elevated protein level $(829 \mathrm{mg} / \mathrm{L})$, but negative cultures for Herpes simplex virus 1 and 2, Varicella zoster virus, Borrelia burgdorferi IgM and IgG and SARS-CoV2.

Whole spinal cord magnetic resonance imaging showed a T2 hyperintense signal abnormality at C3-C6 and D3D7 levels. The cerebral MRI revealed no pathological changes. Electromyography and electroneurography were unremarkable.

The patient was treated with a 5-day course of Methylprednisolone $1 \mathrm{~g}$ per day intravenously. The neurological deficits were constant, therefore the decision to start plasma exchange therapy was made. After 4 sessions of plasma exchange the patient presents with a favourable outcome. The neurological examination revealed a grade 4/5 MRC symmetric paraparesis and hypoesthesia at D6 level. The control whole spine MRI revealed only a T2 hyperintense lesion at D3-D7 level. The urinary catheter had to be kept since urinary outflow was restricted. The patient was discharged clinically stable.

\section{Discussion}

Myelitis describes the inflammation of the spinal cord that could lead to motor, sensory and autonomic impairment [10] with a broad aetiology, notably infectious and systemic autoimmune conditions [11].

Neuromyelitis optica, also known as Devic's disease, is an autoimmune disorder of the CNS characterised by recurrent attacks of longitudinally extensive transverse myelitis and optic neuritis [12]. One of the most important characteristics of NMO-associated transverse myelitis is the extension of the demyelinating lesions, typically involving more than 3 vertebral segments and is centrally distributed [13]. This pathology is associated with serum aquaporin-4 immunoglobulin $\mathrm{G}$ antibodies and positive anti-MOG antibodies [14]. The recurrent involvement of optic nerves has been associated with anti-MOG positive antibodies [15]. Initially, anti-MOG positive antibodies were identified in children with acute disseminated encephalomyelitis (ADEM), but they also could be found in demyelinating disorders or autoimmune diseases [16]. Myelitis is a common manifestation in demyelinating disorders of the CNS and has to be considered into the differential diagnosis between MS and NMO. In MS, demyelinating lesions are confined to less than 3 vertebral segments in length and are distributed more frequently in the lateral columns. Additionally, the presence of oligoclonal bands and elevated IgG index in the CSF constitute criteria for MS diagnosis. [17].
Acute infectious myelitis has different patterns depending on the virus that causes the pathology [18]. The ventral horn pattern was described in infections with flaviviruses (West Nile virus, Dengue virus, Japanese B encephalitis) and enterovirus. The central medullary pattern is seen with Ebstein Barr, Lyme disease and hepatitis. The lateral column is usually affected in infections with T-cell lymphotropic virus-1 and the dorsal column is noted in infections with Varicella zoster virus, neurosyphilis, progressive multifocal leukoencephalopathy (John Cunningham virus), Human immunodeficiency virus [19].

In the presented case, extensive workup revealed no other infectious, inflammatory or demyelinating causes for the patient's transverse myelitis. While the neuropathogenesis of COVID-19 virus is not yet completely understood, two underlying mechanisms are being taken into consideration in our case. Firstly, the neuroinvasive and neurotropic properties of SARS-CoV-2 via the nasal epithelium or vagus nerve through the ACE2-R. This receptor for ACE2 is also found on spinal cord neurons [20]. Secondly, the hyper-inflammatory reaction produced by the virus that activates the cytokine storm, with up-regulation of proinflammatory interleukins (IL) $-2,-6,-7,-10$, tumour necrosis factor alfa causes extensive myelin damage [21].

In our case, SARS-CoV2 was not detected in the CSF sample; this indicates an immune-mediated para-infectious mechanism rather than direct invasion of SARS-CoV 2 into the CNS. This theory is also sustained by the latency of the onset symptoms after the infection with the virus.

The patient tested positive for anti-MOG antibodies. Typically, positivity for anti MOG-antibodies indicates the diagnosis of NMO or NMO-spectrum disorders [22]. The cerebral MRI was unremarkable. Given the clinical and post-infectious context this indicates a humoral autoantibody mediated damage of the CNS [23].

Another hypothesis is that the cytokine storm associated with the infection with SARS-COV 2 triggers an immunemediated CNS aggression [24].

In our case, the Covid-19 para-infectious myelitis appeared in a mild symptomatic patient. The patient's symptoms were consistent with an acute demyelinating spinal lesion, due to the abrupt onset of the flaccid paraparesis, acute urinary retention and D6 level sensory involvement. The spinal MRI confirmed the demyelinating lesions between C3-C6 and D2-D7 with no contrast enhancement. This was confronted against other causes of contrast-enhancing spinal lesions that appear typically in other infectious myelitis such as Epstein-Barr, Herpes Simplex, Varicella Zoster virus, infections with Borrelia Burgdorferi or parasites [25].

Steroidal anti-inflammatory agents, such as high dose methylprednisolone was indicated for our patients, but seeing as the symptoms persistent, the decision was made to start plasma therapeutic exchange (a total of 4 sessions) with clinically and radiologically favourable results. Studies suggest that corticosteroid associated treatment to plasma 
therapy yield better results compared to corticosteroid administration alone [26].

The Covid-19 related myelitis make the diagnosis extremely difficult. Therefore, a differential diagnosis including infectious, inflammatory, primary-demyelinating and paraneoplastic myelitis has to be performed. [27].

\section{Conclusion}

We have described a case of parainfectious myelitis related to the Covid-19 asymptomatic infection. Myelitis is associated with significant morbidity and mortality requiring increased awareness from the clinician. Although we could not find a certain pathophysiology of this disease, we hypothesized an immune-mediated mechanism.

\section{Author's contribution}

MO (Conceptualization, methodology, writing the original draft); $\mathrm{MV}$ (Reviewing, editing, editing the original draft); SA (Reviewing, investigation); BR (Conceptualization, reviewing, editing of the original draft).

\section{Conflict of interest}

None to declare

\section{References}

1. Moreno-Escobar M, Kataria S, Khan E, Subedi R, Tandon M, Peshwe $\mathrm{K}$, et al - Acute transverse myelitis with Dysautonomia following SARSCoV-2 infection: A case report and review of literature. J Neuroimmunol. 2021Apr;353:577523.

2. Wu Y, Xu X, Chen Z, Duan J, Hashimoto K, Liu C, et al - Nervous system involvement after infection with COVID-19 and other coronaviruses. Brain Behav Immun. 2020 Jul;87:18-22.

3. Verstrepen K, Baisier L, Cauwer $\mathrm{H}$ - Neurological manifestations of COVID-19, SARS and MERS. Acta Neurol Belg. 2020 July;1-10.

4. Lewis A, Frontera J, Placantonakis D, Lighter J, Galetta S, Balcer L, et al - Cerebrospinal fluid in COVID-19: A systematic review of the literature. J Neurol Sci. 2021 Feb;421:117316.

5. Advani S, Zali A, Ommi D, Fatemi A, Khoshnoud J, Ashrafi F - Transverse myelitis in COVID-19 patients: Report of two cases. Research Square. 2020 Nov; 10.21203

6. Durrani M, Kucharski K, Fien S - Acute Transverse Myelitis Secondary to Severe Acute Respiratory Syndrome Coronavirus 2 (SARS-CoV-2): A Case Report. Clin Pract Cases Emerg Med. 2020 Aug;4(3):344-348.

7. Espindola O, Brandao C, Gomes Y, Siqueira M, Soares C, Lima M, et al -Cerebrospinal fluid findings in neurological diseases associated with COVID-19 and insights into mechanisms of disease development. Int J Infect Dis. 2021 Jan;102:105-162.
8. Ali L, Khan A, Elalamy O, Canibano B, Al hatou M, Ghulam A, et al - A rare presentation of acute flaccid myelitis in covid-19 patient: a case report. PJNS. 2020 Jul; 15(3).

9. Abdelhady M, Elsotouhy A, Vattoth S, MBBCh, FRCR, DABR, et al - Acute flaccid myelitis in COVID-19. BJR Case Rep. 2020 Sep;6(3):20200098.

10. Chakraborty U, Chandra A, Ray A, Biswas P - COVID-19-associated acute transverse myelitis: a rare entity. BMJ Case Reports CP 2020;13:e238668.

11. Jarius S, Wildemann B - Devic's disease before Devic: Bilateral optic neuritis and simultaneous myelitis in a young woman (1874). Journal of the Neurological Sciences. 2015 Aug;358(1-2):419-421.

12. Wu Y, Zhong L, Geng J - Neuromyelitis optica spectrum disorder: Pathogenesis, treatment, and experimental models. Mult Scler Relat Disord. 2019 Jan;27:412-418.

13. Kitley J, Leite M, George JS, Palace J - The differential diagnosis of longitudinally extensive transverse myelitis. Mult Scler. 2012 Mar;18(3):271-85

14. Lana-Peixoto A, Talim N - Neuromyelitis Optica Spectrum Disorder and Anti-MOG Syndromes. Biomedicines. 2019 Jun;7(2):42.

15. Asnafi S, Morris P, Sechi E, Pittock S, Weinshenker B, Palace J, et al - The frequency of longitudinally extensive transverse myelitis in MS: A population-based study. Mult Scler Relat Disord. 2020 Jan;37:101487.

16. Jobling K, Ledingham D, Guadagno J - Positive anti-MOG antibodies in a patient with Sjogren's syndrome and transverse myelitis. Eur $J$ Rheumatol. 2019 Apr;6(2):102-104.

17. Asundi A, Cervantes-Arslanian A, Lin N, Barbosa F - Infectious Myelitis. Semin Neurol. 2019 Aug;39(4):472-481.

18. Mihai C, Jubelt B - Infectious Myelitis. Curr Neurol Neurosci Rep. 2012 Dec;12:633-641.

19. Aguilo-Gordo D, Flores-Barragan M, Ferragut-Loret F, Guzman V Acute myelitis and SARS-CoV-2 infection: A new etiology of myelitis? J Clin Nerosci. 2020 Oct:80:280-281.

20. West T, Hess C, Cree B - Acute Transverse Myelitis: Demyelinating, Inflammatory, and Infectious Myelopathies. Semin Neurol. 2012 Apr;32(2):97-113.

21. Reindl M, Waters $P$ - Myelin Oligodendrocytes glycoprotein antibodies in neurological disease. Nat Rev Neurol. 2019 Feb;15(2):89-102.

22. Peschl P, Bradl M, Hoftberger R, Reindl M - Myelin Oligodendrocyte Glycoprotein: Dechiphering a Target in Inflammatory Demyelinating Diseases. Front Immunol. 2017; 8: 529.

23. Rodriguez de Antonio L, Gonzalez-Suarez I, Perez M, FernandezBarriuso I - Para-infectious anti-GD2/GD3 IgM myelitis during the Covid-19 pandemic: Case report and literature review. Mult Scler Relat Disord. 2021 Apr;49:102783.

24. Chow, CCN, Magnussen, J, Ip, J, Su, Y - Acute transverse myelitis in COVID-19 infection. BMJ Case Report CP. 2020;13:e236720.

25. Novi, G, Rossi, T, Pedemonte, E, Saitta, L, Rolla C, Roccatagliata L, et al - Acute disseminated encephalomyelitis after SARS-CoV-2 infection. Neurol Neuroimmunol Neuroinflamm. 2020 Sep;7(5)e797.

26. Balasa R, Maier S, Bajko Z, Motataianu A, Crisan A, Balasa A Longitudinally extensive transverse myelitis in neuromyelitis optica: a prospective study of 13 Caucasian patients and literature review. Acta Neurol Belg. 2015 Dec;115(4):635-42.

27. Roy D, Ghosh R, Dubey S, Ray K - Neurological and neuropsychiatric impacts of COVID-19 pandemic. Can J Neurol Sci. 2020 Aug;5:1-16. 